\title{
Improved Hybrid DLBS Artificial Bee Colony Optimization Algorithm based on Parallel Computing Environment
}

\author{
Bhuvnesh Pathania \\ M.Tech. (CSE), Pursuing \\ Department of Computer Science \& Engineering, \\ Vaishno College of Engineering, Himachal \\ Pradesh.
}

\author{
Abhilash Sharma \\ Head \& Assistant Professor \\ Department of Computer Science \& Engineering, \\ Vaishno College of Engineering, Himachal \\ Pradesh.
}

\begin{abstract}
This paper represents the Parallel Computing is now extremely popular because of its wide variety of applications through internet. The particular service based approaches which are aware from the server selection through the parallel can easily progress toward the cost and performance of parallel computing. A new hybrid DLBS Artificial bee colony optimization algorithm for parallel computing environment has been done. The overall objective of this paper is enlighten the performance analysis on dynamic load balancing strategy (DLBS) with $\mathrm{ABC}$ algorithm which enhances the results further by using mean flow time parameter.
\end{abstract}

\section{Keywords}

Parallel computing, Scheduling System, DLBS, Ant Colony optimization, Artificial Bee Colony

\section{INTRODUCTION}

A computing system is described as collection of either homogeneous systems or heterogeneous systems. During an instruction cycle, execution of programmed on computing system may use different number of processors at different instance of time [8]. Emerging concept that can simultaneously execute various tasks on different processors is known as processing. It is an effective way of solving complex and computation intensive issues. System can be categorized into two systems: homogeneous and heterogeneous, depending upon the nature of processors [11]. The core component of system is multiprocessor system (MTS). In MTSs, there are multiple input/output modules, multiple processing elements, and multiple memory modules. Computing is some sort of kind of working out through which quite a few measurements or even the rendering of techniques are executed simultaneously. Significant troubles are often separated into smaller sized versions that will then possibly be sorted out all at once [15]. The core component of system is multiprocessor system (MTS) [14, 15]. In MTSs, there are multiple input/output modules, multiple processing elements, and multiple memory modules. Computing is some sort of style of formula wherein a lot of estimations or perhaps the setup of methods are finished simultaneously. Big troubles are frequently broken into small types, which will are able to possibly be fixed on a similar time. There are numerous many forms with computing: bit-level, instruction-level, files, and taskism.ism has become currently employed for a long time, generally in high-performance computing, although desire inside has expanded of late a result of the real difficulties preventing scaling[13].

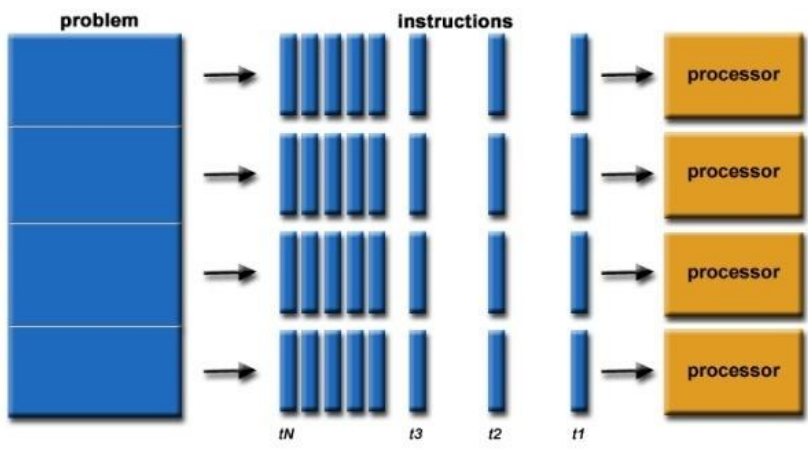

Fig 1: Parallel Computing

\subsection{Scheduling System}

A schedule is surely an part involving technique sources to help person work opportunities for several moment periods. As a result, an agenda might be explained by furnishing everyday circumstances wherever a big difference involving sources part takes place so long as often this modification can be initiated simply by organizing technique or even the organizing method is alerted involving this modification [13]. The particular organizing technique of an multiprocessor gets a flow involving work submissions details as well as generates a legitimate schedule. All of us take advantage of the period 'flow'to point of which submissions details for various work opportunities do not need to go through the identical time. As well the appearance involving any sort of details are certainly not foreseen, that is definitely, this organizing technique might not exactly keep in mind every details returning within the future. As a result, this organizing technique will have to handle named 'on-line' behavior. Scheduling is the allotment of assets to execute a accumulation of jobs [14] and has broad range of usage in entire economic field in present reality situation. Scheduling problems constantly come up in several trade situations a well known as transportation, information processing.

\subsubsection{Taxonomy of Scheduling Algorithm}

A task is the unit of computation in our computing systems, and several tasks working towards a common goal are called a job [2]. There are two levels of scheduling in multiprocessor system:

1. Global scheduling

2. Local scheduling. 
1. Local scheduling:-Determines which of the set of variables tasks at a processor runs next on that processor [4].

2. Global scheduling: - Involves assigning a task to a particular processor within the system. This really is also referred to as applying, job position, along with complimenting [2, 3]. International organizing normally takes spots ahead of neighborhood organizing, whilst job migration, and also active reassignment, can alter the international applying through switching an undertaking to an alternative processor. To help move an undertaking, the device freezes the work, spend less their point out, exchange this preserved point out to an alternative model, along with reboot this task. There may be important cost to do business linked to migration your jogging task.

\section{DYNAMIC LOAD BALANCING STRATEGY (DLBS )}

The performance analysis algorithm is known as dynamic load balancing strategy (DLBS) for hypercube interconnection network. It is dynamic in the sense that no prior information of the load is assumed. DLBS algorithm takes multiple numbers of tasks to provides least load imbalance and therefore, IF is lesser. The benefit of DLBS algorithm is that it minimizes the execution time and LIF even for large number of tasks in homogeneous system and applies on hypercube (HC) network. $\mathrm{HC}$ is constructed by linking every node to a node utmost from it. The number of nodes, degree and diameter in $\mathrm{HC}$ network is $2 n, n$ and $n$ respectively.

\section{DLBS algorithm}

1. Generate random tasks

2. Scheduler calculates the load on each processor, total load and ideal load

3. Finds the maximum overloaded (MOL) and maximum under loaded (MUL) processor

4. Checks connectivity between MOL and MUL in hypercube network

5. Starts execution time.

6. Migration of load takes place from MOL to MUL processor

7. Repeats steps 3-6 until processors become moderate.

8. Ends execution time.

\section{METAHEURISTIC TECHNIQUES}

\subsection{ABC Algorithm}

The Artificial bee colony Algorithm is a metaheuristic technique which is proposed in 2005.It is actually inspired by the brilliant foraging behavior of honey bees. In this Algorithm colony comprises three kind of artificial bees i.e. employed bees, onlooker bees and Scout bees.

4 Employed Bee: Employed bee try to identify new source for food near the old source. If nectar amount is greater in new food source than old food source than detected source is memorized. Employed bees share their information with onlooker bee via wangle dance.

5 Onlooker Bee: Onlooker bees saw the movements of dance of employed bees and then select one of the food source based on their dance and afterward goes to that food source. After selecting a neighbor near to that food source, it evaluates their nectar quantity. An onlooker bee evaluates the knowledge received through the employed bees and then tries to get a new food source within the circle of selected food depending on the following examined information.

6 Scout Bees: The Scout bees are not predetermined in the colony. Scout bees are generally converted from some employed bees which usually abandon from their particular food resources by using fixed amount of rounds. The parameter 'Limit' control number of scout bee. If predefined Limit number could not improve food source then its value is randomly generated again.

7 In $\mathrm{ABC}$ algorithm food source and nectar amount shows the solution for optimization problem and quality of solution respectively. Every food source is related to a particular employed bee inside colony. The amount of food sources is equivalent to amount of employed bees which in turn equal to onlooker bees. Thus amount of food sources is $50 \%$ of the population [12].

\subsection{Ant Colony Optimization Algorithm}

The ACO algorithm is animated by the scrounging behavior of ants; especially the pheromone interaction between ants with regard to a better path amongst colony and a food originates in an environment. This process is known as stigmergy. The main purpose is to feat heuristic and historical data so as to build up candidate solutions plus fold the data found out from building results into account. No. of solutions have been build up but only one distinct piece at once in the probabilistic step-by-step manner. The probability with which a component is picked out is decided by heuristic part of an element and the solution's quality out of which the element has been known with respect to history is included. History is modified relative to the tone of most beneficial recognized solution and is diminished relative to the use of distinct components of solution.

Pseudo Code for ACO:

1. Procedure ACO_Metaheuristic

2. While (not_termination)

$$
\begin{aligned}
& \text { a. GenerateSolutions () } \\
& \text { b. DaemonActions () } \\
& \text { c. Pheromone Update () }
\end{aligned}
$$

\section{End While \\ 4. End Procedure}

\section{RELATED WORKS}

Joanna Kołodziej et al. [1] proved the performance of proposed method within the minimization of power usage by entire system as well as in variable load balancing belonging to the resources within grid clusters that are enough to manage required Quality grade. Marfa Arsuaga-Rfos et al. [2] presented Multi Objective Artificial bee colony (MOABC) Optimization Algorithm which optimized both execution time as well as power usage to solve job scheduling issues within. Mohammad Shojafar et al. [3] introduced hybrid technique known as FUGE which is dependent on fuzzy principle and genetic algorithm which is designed to execute optimum balancing of loads taking in to account execution time as well as cost. Hongze Qiu et al. [4] offered a replication upon approach to succeed in many different goals, like lowering the running time and power cost. The primary algorithms employed are actually genetically 
protocol and also ant Colony optimization. Saeed Javanmardi1, et al. [5] represented a new hybrid job Scheduling Methodology using genetic Algorithm with fuzzy theory which often takes into account the load balancing of system plus reduces total running time and cost. Jing Liu et al. [6] introduced model for scheduling, the resolution solution dependent on multi-objective genetic algorithmic rule (MOGA) is created together with the research aimed at guidelines, crossover operators, choice operators as well as approach to selecting parallel solutions. Fan Zhang et al. [7] introduced the ordinal optimization technique from a single goal to multiple goals having a vectorized ordinal optimization (VOO) method. It demonstrated the sub optimality via mathematical analysis. F. Farahnakian et al. [8] presented architecture of distributed systems to execute a VM Consolidation dynamically to minimize power usage for data centers of parallel and also keep the specified QoS. Since VM Consolidation issue is a totally NP hard. Mohammad Masdari et al. [9] detailed analysis of workflow scheduling schemes for scheduling workflows either simple or scientific proposed for parallel computing environment. It focuses on giving solution to the issues that is, proper execution of workflows by taking into account QOS requirements e.g. budget constraints and deadline. Javid Taheri et al. [10] works on matchmaking scheduling phase and provide two algorithms to reduce the make-span for executing all jobs and transfer time for all data-fields. It use two collaborating algorithm for schedule job \& replicate data-fields to connected nodes and storage nodes respectively. Erkan Besdok et al. [11] presented Comparison and analysis of four algorithm DLBS-search (CK), Particle swarm optimization (PSO), Differential evolution (DE) and artificial bee colony (ABC) algorithms with each other. The PSO algorithm is successful in the solution of many benchmark functions, but its well-known stability problem restricts the success rate of this algorithm against the $\mathrm{CK}$ and DE algorithms. Fabrizio Ferrandi et al. [13] presented a technique is provided which is based on ant colony optimization technique. It efficiently solves the multiple scheduling. It reduces the total execution time. It provides mapping of task and communication. Zhang $\mathrm{Y}$, et al. [16] proposed a hybrid scheduling mechanism and a two-step rescheduling approach. In former, suitable resources are selected statically based on resource's aggregated computing power followed by scheduling each task dynamically on the selected resources. Frédéric Pinel et al. [17] presented the sensitivity analysis of a Cellular Genetic Algorithm (CGA) with local search is used to design a new and faster heuristic for the problem of mapping independent tasks to a distributed system. Saurabh Bilgaiyan et al. [18] presented a study of various swarm-based and evolutionary optimization techniques effectively used for task-resource mapping and scheduling on parallel computing systems. M. Prakash et al. [19] introduced DLBS optimization algorithm for optimum job allotment associated with resources upon every node. DLBS optimization algorithm selects the suitable source out of accessible resources. This algorithm assigns all the jobs efficiently from taking into consideration the timeline condition belonging to the end users and also the very low running time. Xiaoli Wang et al. [20] focused on how to reduce the energy consumption of data center thereby increasing energy efficiency of servers. The main objective includes improving energy efficiency of servers by properly using allocation and scheduling strategies. To achieve the desired goal, the relation between server's performance and energy consumption is taken into consideration, using which another energy-aware scheduling optimization model is prepared which is based on the Map Reduce. S. Lorpunmanee et al.[21] addressed the issue by simply acquiring a standard platform associated with grid scheduling utilizing dynamic data as well as an colony optimization that enhance the efficient scheduling. The particular efficiency of numerous rules for instance First Come First Serve,(FCFS), Earliest Due Date (EDD), Earliest Release Date (ERD), and an Ant Colony Optimization (ACO) are compared. Mahfooz Alam et al. [22] discussed for the powerful download controlling within multiprocessor system. Fill controlling is actually the strategy regarding splitting the entire download one of several processor chips of your handed out procedure for you to success task's effect period and also learning resource use while neglecting an ailment where by number of processor chips are usually full or maybe underneath jam-packed or maybe somewhat loaded.

\section{GAPS IN LITERATURE}

Following are the various gaps in earlier work [22] :-

1. The use of DLBS search and Ant colony optimization has demonstrated a minimum convergence rate to the true global minimum even at large numbers of dimensions.

2. Artificial bee colony optimization algorithm has been highly accepted as a global optimization algorithm of current scenarios for distributed optimization and control.

3. Artificial bee colony optimization algorithm has already drawn the interest of researchers due to its efficiency in resolving real-world optimization issues arising in numerous application domains.

\section{METHODOLOGY AND RESULTS}

A) Proposed Methodology

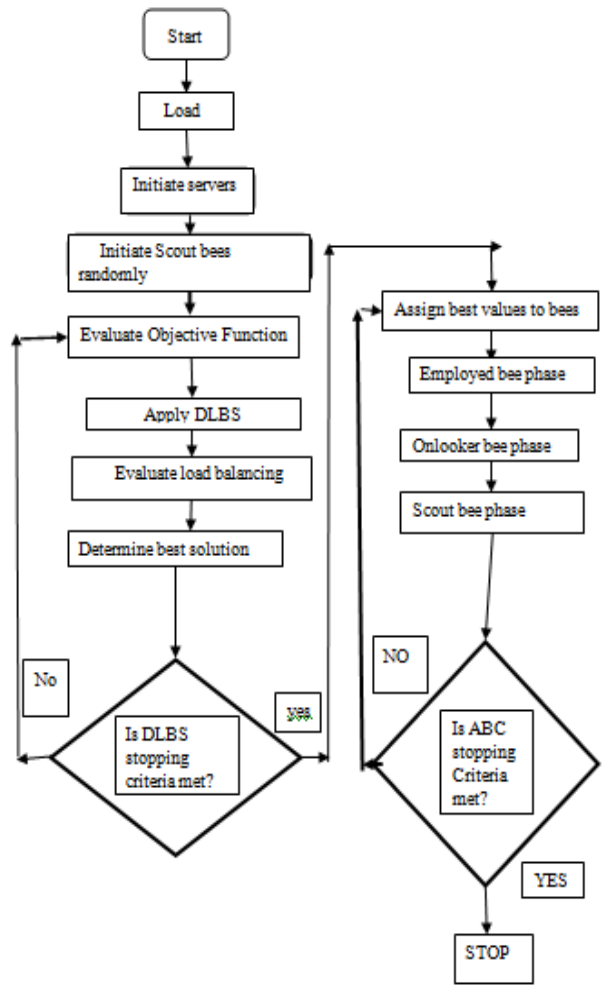

Fig 2: Flowchart of the proposed technique

B) Experimentation

This section provides cross validation and verification of existing deterministic techniques i.e. First Come First Serve Scheduling (FCFS), Longest Job First Scheduling (LJFS), 
Shortest Job First Scheduling (SJFS) and Stochastic i.e. Hybrid of ACO \& DLBS and proposed techniques i.e. ABC \& DLBS with respect to some well-defined performance evaluation criteria The proposed method is evaluated over different performance metrics such as mean Flow Time with respect to number of jobs and number of servers in comparison to existing technique.

\section{Mean Flow Time}

The mean flow time of a schedule gives a way of measuring of the average time period which a job require inside a computer system as well as the average number of incomplete jobs in the system. It can also described as the sum of the completion times of all jobs within the system.

\section{Mean Flow Time}

Total processing time + total waiting time Number of jobs

Table No. 1 Mean Flow Time Using different Scheduling techniques when no. of servers $=10$

\begin{tabular}{|c|c|c|c|c|c|}
\hline $\begin{array}{c}\text { No. of } \\
\text { jobs }\end{array}$ & LJFS & FCFS & SJFS & DLBS & $\begin{array}{c}\text { ABC+DLBS } \\
\text { (proposed) }\end{array}$ \\
\hline 100 & 320 & 291 & 214 & 202 & 153 \\
\hline 200 & 940 & 956 & 667 & 557 & 489 \\
\hline 300 & 2271 & 1652 & 1470 & 1151 & 1068 \\
\hline 400 & 3760 & 2630 & 2011 & 1782 & 1698 \\
\hline 500 & 5569 & 4283 & 3226 & 2810 & 2718 \\
\hline 600 & 8480 & 7106 & 4413 & 3990 & 3878 \\
\hline 700 & 10448 & 8291 & 5376 & 5205 & 5089 \\
\hline 800 & 15120 & 9188 & 7468 & 6737 & 6643 \\
\hline 900 & 16540 & 13404 & 9771 & 8856 & 8692 \\
\hline 1000 & 24462 & 16059 & 12270 & 9482 & 9334 \\
\hline
\end{tabular}

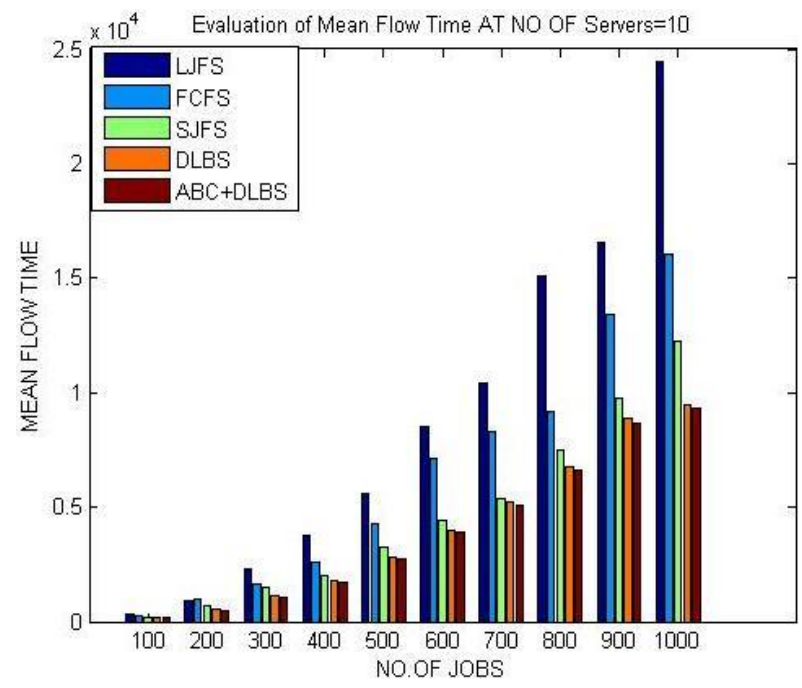

Figure no. 3 Evaluation of Mean Flow Time on the basis of server
Table no. 2 mean flow time using different Scheduling techniques when no. of jobs $=\mathbf{5 0 0}$

\begin{tabular}{|c|c|c|c|c|c|}
\hline $\begin{array}{c}\text { No. of } \\
\text { servers }\end{array}$ & LJFS & FCFS & SJFS & DLBS & $\begin{array}{c}\text { ABC+DLB } \\
\text { S } \\
\text { (proposed) }\end{array}$ \\
\hline 10 & 4089 & 3907 & 3265 & 3062 & 2980 \\
\hline 15 & 3051 & 2871 & 2104 & 1942 & 1889 \\
\hline 20 & 2700 & 2319 & 1600 & 1420 & 1318 \\
\hline 25 & 2137 & 1673 & 1381 & 1253 & 1180 \\
\hline 30 & 1914 & 1568 & 1140 & 1048 & 922 \\
\hline 35 & 1680 & 1313 & 1035 & 929 & 851 \\
\hline 40 & 1410 & 1130 & 921 & 804 & 708 \\
\hline 45 & 1376 & 1028 & 817 & 740 & 638 \\
\hline 50 & 1176 & 970 & 765 & 691 & 603 \\
\hline
\end{tabular}

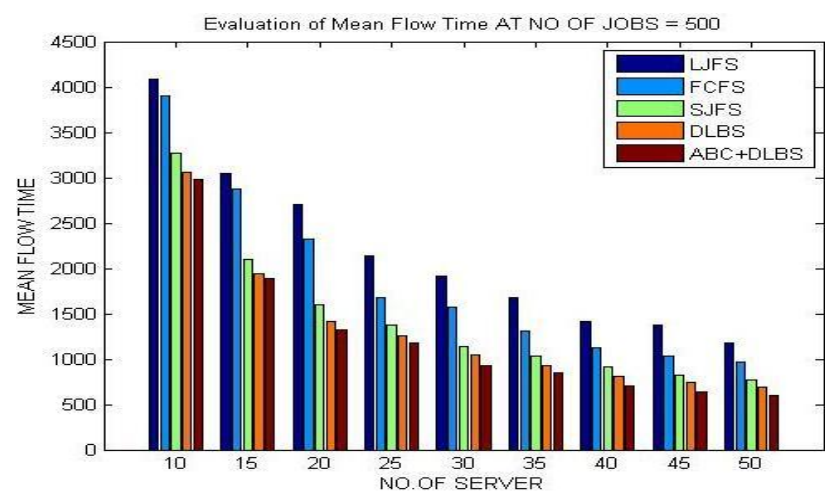

Figure No.4 Evaluation of Mean Flow Time on the basis of jobs

Table no. 1 and 2 clearly shows that mean flow time in both cases as on the basis of jobs and servers is minimum in case of proposed Algorithm i.e. hybrid of ABC \& DLBS than existing one.

\section{CONCLUSION}

With the increasingly ubiquitous nature of parallel computing, users are starting to explore new ways to interact with, and exploit these developing paradigms. In this paper the use of DLBS search and Ant colony optimization has shown a low convergence rate to the true global minimum even at high numbers of dimensions. Artificial bee colony optimization algorithm has been widely accepted as a global optimization algorithm of current interest for distributed optimization and control. Artificial bee colony optimization algorithm has already drawn the attention of researchers because of its efficiency in solving real-world optimization problems arising in several application domains. The evaluation of technique is simple and effective is done using parameter like Mean flow time. This comparison has shown that the proposed work results are much better than the existing results. This is because the reduction in mean flow time is 101 .

\section{REFERENCES}

[1] Wang, Lizhe, et al. "Energy-aware parallel task scheduling in a cluster." Future Generation Computer Systems 29.7 (2013): 1661-1670.

[2] Arsuaga-Ríos, María, and Miguel A. Vega-Rodríguez. "Energy optimization for task scheduling in distributed 
systems by an Artificial Bee Colony approach." Nature and Biologically Inspired Computing (NaBIC), 2014 Sixth World Congress on. IEEE, 2014.

[3] Javanmardi, Saeed, et al. "Hybrid job scheduling algorithm for cloud computing environment." Proceedings of the Fifth International Conference on Innovations in BioInspired Computing and Applications IBICA 2014. Springer International Publishing, 2014.

[4] Zhao, Jianfeng, and Hongze Qiu. "Genetic algorithm and ant colony algorithm based Energy-Efficient Task Scheduling." Information Science and Technology (ICIST), 2013 International Conference on. IEEE, 2013.

[5] Javanmardi, S., Shojafar, M., Amendola, D., Cordeschi, N., Liu, H., \& Abraham, A. (2014). Hybrid job scheduling algorithm for cloud computing environment. In Proceedings of the Fifth International Conference on Innovations in Bio-Inspired Computing and Applications IBICA 2014 (pp. 43-52). Springer International Publishing.

[6] Liu, J., Luo, X. G., Zhang, X. M., Zhang, F., \& Li, B. N. (2013). Job scheduling model for cloud computing based on multi-objective genetic algorithm. IJCSI International Journal of Computer Science Issues, 10(1), 134-139.

[7] Liu, S. L., Liu, Y. X., Zhang, F., Tang, G. F., \& Jing, N. (2007). Dynamic web services selection algorithm with QoS global optimal in web services composition. Ruan Jian Xue Bao(Journal of Software), 18(3), 646-656.

[8] Farahnakian, F., Ashraf, A., Pahikkala, T., Liljeberg, P., Plosila, J., Porres, I., \& Tenhunen, H. (2015). Using ant colony system to consolidate vms for green cloud computing. IEEE Transactions on Services Computing, 8(2), 187-198.

[9] Masdari, M., ValiKardan, S., Shahi, Z., \& Azar, S. I. (2016). Towards workflow scheduling in cloud computing: A comprehensive analysis. Journal of Network and Computer Applications, 66, 64-82.

[10] Dorronsoro, B., Nesmachnow, S., Taheri, J., Zomaya, A. Y., Talbi, E. G., \& Bouvry, P. (2014). A hierarchical approach for energy-efficient scheduling of large workloads in multicore distributed systems. Sustainable Computing: Informatics and Systems, 4(4), 252-261.

[11] Civicioglu, Pinar, and Erkan Besdok. "A conceptual comparison of the Cuckoo-search, particle swarm optimization, differential evolution and artificial bee colony algorithms." Artificial intelligence review 39.4 (2013): 315-346.

[12] Sajedi, Hedieh, and Maryam Rabiee. "A metaheuristic algorithm for job scheduling in grid computing." International Journal of Modern Education and Computer Science 6.5 (2014): 52.

[13] Ferrandi, F., Lanzi, P. L., Pilato, C., Sciuto, D., \& Tumeo, A. (2010). Ant colony heuristic for mapping and scheduling tasks and communications on heterogeneous embedded systems. IEEE Transactions on ComputerAided Design of Integrated Circuits and Systems, 29(6), 911-924.

[14] Alkhanak, Ehab Nabiel, Sai Peck Lee, and Saif Ur Rehman Khan. "Cost-aware challenges for workflow scheduling approaches in cloud computing environments: Taxonomy and opportunities." Future Generation Computer Systems 50 (2015): 3-21.

[15] Li, Jun-Qing, Quan-Ke Pan, and Kai-Zhou Gao. "Paretobased discrete artificial bee colony algorithm for multiobjective flexible job shop scheduling problems." The International Journal of Advanced Manufacturing Technology 55.9-12 (2011): 1159-1169.

[16] Jia, H. Z., Nee, A. Y., Fuh, J. Y., \& Zhang, Y. F. (2003) A modified genetic algorithm for distributed scheduling problems. Journal of Intelligent Manufacturing, 14(3-4), 351-362.

[17] Pinel, F., Dorronsoro, B., Pecero, J. E., Bouvry, P., \& Khan, S. U. (2013). A two-phase heuristic for the energyefficient scheduling of independent tasks on computational grids. Cluster Computing, 16(3), 421-433.

[18] Bilgaiyan, Saurabh, Santwana Sagnika, and Madhabananda Das. "An analysis of task scheduling in cloud computing using evolutionary and swarm-based algorithms." International Journal of Computer Applications 89.2 (2014)

[19] Priya, S. Baghavathi, M. Prakash, and K. K. Dhawan. "Fault tolerance-genetic algorithm for grid task scheduling using check point." Grid and Cooperative Computing, 2007. GCC 2007. Sixth International Conference on IEEE, 2007.

[20] Li, S., Li, G., Wang, X., \& Liu, Q. (2005). Minimizing makespan on a single batching machine with release times and non-identical job sizes. Operations Research Letters, 33(2), 157-164.

[21] Lorpunmanee, S., Sap, M. N., Abdullah, A. H., \& Chompoo-inwai, C. (2007). An ant colony optimization for dynamic job scheduling in grid environment. International Journal of Computer and Information Science and Engineering, 1(4), 207-214.

[22] Bokhari, M. U., Mahfooz Alam, and Faraz Hasan. "Performance analysis of dynamic load balancing algorithm for multiprocessor interconnection network." Perspectives in Science 8 (2016): 564-566. 